\title{
Vasovagal syncope in 2016: the current state of the faint
}

In this article, we will review the challenges in defining syncope and the evolution of its definition over the past three decades. We will then highlight key recommendations from the HRS 2015 consensus document on the investigation and management of VVS, as well as discuss current gaps in our understanding of VVS.

\section{The evolving definition of syncope}

The term "syncope" suggests a clinical and intuitively grasped picture, yet it has been inconsistently and infrequently defined in the literature. Table 1 review some of the syncope definitions over the past couple decades.

Originally, syncope was defined as a transient state of unconsciousness characterized by spontaneous recovery, or recovery in a supine position [1]. This definition was developed for research purposes to describe tilt table test outcomes, and was not intended for clinical use. Subsequently, the European Society of Cardiology (ESC) published their first guidelines for the management of syncope in 2001, which were updated in 2004 [2]. The ESC defined syncope as a transient loss of consciousness attributable to global cerebral hypoperfusion, and characterized by rapid onset, brevity, and spontaneous recovery. In 2009, the ESC published their updated guidelines for the diagnosis and management of syncope [3]. One significant update was highlighting the definition of syncope in the broader context of transient loss of consciousness (T-LOC). The term T-LOC was coined to encompass all disorders characterized by a self- limited loss of consciousness, irrespective of mechanism. For example, T-LOC includes syncope, epileptic seizures, psychogenic pseudosyncope, and other miscellaneous causes. Syncope is differentiated from other forms of T-LOC due to its unique pathophysiology of transient global cerebral hypoperfusion.

The ESC definition of syncope provided a more specific and pathophysiological basis for the diagnosis of syncope, and sought to clarify the confusion with the broader T-LOC umbrella. While a laudable effort, the inclusion of "global cerebral hypoperfusion" in the definition proved challenging for clinicians to apply during their initial encounter with afflicted patients. The definition could only be applied strictly with the demonstration of cerebral hypoperfusion, again leaving clinicians without a widely applicable working definition.

In September 2013, a multispecialty workshop of North American and European syncope experts met in Gargnano, Italy with the aim of obtaining consensus on the optimal evaluation and management of syncope patients in the emergency department. The Gargnano consensus conference removed the physiologic criterion for syncope, and substituted a criterion that would exclude other causes of loss of consciousness [4]. Syncope was defined as "a transient loss of consciousness, associated with inability to maintain the postural tone and with immediate spontaneous and complete recovery, associated with clinical features suggestive of specific forms of syncope (e.g. vasovagal, orthostatic, cardiac); or the absence of clinical features specific for another form of transient loss of consciousness such as epileptic seizure, hypoglycemia, or trauma". This was a practical, feasible approach that after vigorous debate met agreement by cardiologists, neurologists, internists, family
Derek S Chew, Satish R Raj and Robert S Sheldon*

Department of Cardiac Sciences, Libin Cardiovascular Institute of Alberta, University of Calgary, Calgary, AB, Canada

*Author for correspondence:

Tel.: +4032208191

sheldon@ucalgary.ca 
doctors, and emergency department physicians.

In 2015, the Heart Rhythm Society (HRS), in collaboration with various international professional societies, released an expert consensus document on three common syndromes: Postural Tachycardia Syndrome (POTS), Inappropriate Sinus Tachycardia (IST), and Vasovagal Syncope (VVS) [5]. The three primary objectives of this document were to establish working criteria for the diagnosis of these conditions (i.e. provide definitions that were both useful at the bedside as well as provided uniform inclusion criteria for research studies), provide recommendations for the assessment and management of these disorders, and identify the main areas for further collaborative research.

The HRS document minimally modified the Gargnano definition of syncope, and reads "A transient loss of consciousness, associated with inability to maintain postural tone, rapid and spontaneous recovery, and the absence of clinical features specific for another form of transient loss of consciousness such as epileptic seizures". This is consistent with the ESC Syncope 2009 definition. Most clinicians do feel that the mechanism of syncope involves cerebral hypo perfusion, but this cannot usually be proven at the point of care. The HRS definition is designed to be useable in the clinic or the emergency department setting.

\section{Vasovagal Syncope: Overcoming the Diagnostic Challenge}

The HRS document also provides a consensus definition of vasovagal syncope, the most common cause of syncope, based on a mix of published evidence and expert opinion. It defines VVS as a syncope syndrome that usually 1) occurs with upright posture greater than 30 seconds, or with exposure to emotional stress, pain, or medical settings; 2) features diaphoresis, warmth, nausea, and pallor; 3) is associated with hypotension and relative bradycardia, when known; and 4) is followed by fatigue.

The consensus document importantly highlights that the key to an accurate diagnosis of VVS lies within the clinical history. There are 4 categories of key diagnostic features: predisposing situations, prodromal symptoms, physical signs, and recovery time and symptoms.

Vasovagal faints usually occur after prolonged standing or with sitting (with the legs down), but can be triggered even in the supine position by exposure to medical or dental situations, pain, or scenes of injury. The term "prolonged" can mean as little as 2 to 3 minutes, and is an important feature differentiating VVS from both initial and initial orthostatic hypotension (in which the blood pressure drops within the first minute). Prodromal features include progressive pre syncope, diaphoresis, a sense of warmth or flushing, nausea or abdominal discomfort, and visual blurring or frank loss of vision. While unconscious, patients are usually motionless, but myoclonic movements can be noted by casual observers (in up to $10 \%$ ), which can lead to a misdiagnosis of seizures [6]. Unconsciousness usually lasts less than 1 to 2 minutes, but full recovery can be delayed: patients often feel very tired minutes to hours following a syncopal spell.

With a careful and focused history, the diagnosis of vasovagal syncope can be made correctly without further investigation. However, further investigation may be helpful in specific circumstances and certain populations. For example, tilt table testing may be useful in some patients, such as those 1) with suspected VVS but lacking clear diagnostic features, 2) those who may have either convulsive syncope or true seizure activity, and 3) those who might have pseudosyncope. Tilt table testing features prolonged passive postural stress to establish whether patients have an autonomic substrate for vasovagal syncope. It is important to note that tilt testing when positive suggests a predisposition to vasovagal syncope, but does not establish it as the etiology of the patient's syncope. In other words, a positive tilt table test may contribute to the diagnosis of VVS, but it does not provide a diagnosis of VVS by itself.

The HRS consensus document also clarifies the role for prolonged electrocardiographic monitoring, such as implantable loop recorders (ILRs). These ILRs contain loop memory, continuously records and deletes ECGs, and stores the ECGs when activated by the patient after a syncopal episode. The recorded ECG can help provide insight into the etiology of syncope. For example, the simultaneous suppression of both AV and sinus node activity strongly suggests a diagnosis of VVS [7]. However, a syncopal episode associated with normal sinus rhythm may be due to several disorders such as orthostatic hypotension, vasovagal or carotid sinus reflexes, or even psychogenic pseudosyncope. ILRs can be considered for elderly patients (7th and 8 th decades) with recurrent and troublesome syncope; however, they have only been shown to improve care in a subset of patients with VVS who are older, have documented asystole, and a negative tilt test. These patients appear to benefit from permanent pacing [8].

\section{Managing Vasovagal Syncope}

Table 2 outlines the 2015 HRS expert consensus recommendations for management of VVS. It is worth noting the bare handful of recommendations for management with clear benefit to risk ratio (class I and class IIa). Although various lifestyle and medication strategies have had favourable results in uncontrolled 


\begin{tabular}{|c|c|c|}
\hline Year, Source & Definition & Reference \\
\hline 1992, Tilt Table Study & $\begin{array}{l}\text { A transient state of unconsciousness characterized by spontaneous recovery, } \\
\text { or recovery in a supine position }\end{array}$ & [1] \\
\hline $2004 \& 2009$, ESC & $\begin{array}{l}\text { A transient loss of consciousness attributable to global cerebral } \\
\text { hypoperfusion, and characterized by rapid onset, brevity, and spontaneous } \\
\text { recovery }\end{array}$ & {$[2,3]$} \\
\hline $\begin{array}{l}\text { 2013, Gargnano } \\
\text { Conference }\end{array}$ & $\begin{array}{l}\text { A transient loss of consciousness, associated with inability to maintain the } \\
\text { postural tone and with immediate spontaneous and complete recovery, } \\
\text { associated with at least } 1 \text { of the following: (1) clinical features suggestive } \\
\text { of specific forms of syncope (eg. vasovagal, orthostatic, cardiac); or (2) the } \\
\text { absence of clinical features specific for another form of transient loss of } \\
\text { consciousness such as epileptic seizure, hypoglycemia, or trauma. }\end{array}$ & [4] \\
\hline 2015, HRS & $\begin{array}{l}\text { A transient loss of consciousness, associated with inability to maintain } \\
\text { postural tone, rapid and spontaneous recovery, and the absence of clinical } \\
\text { features specific for another form of transient loss of consciousness such as } \\
\text { epileptic seizures. }\end{array}$ & [5] \\
\hline
\end{tabular}

Table 2: HRS 2015 Recommendations for Management of VVS.

Lifestyle and Medical Treatment for Vasovagal Syncope

\begin{tabular}{|c|c|c|}
\hline & Class & Level \\
\hline $\begin{array}{l}\text { Education, reassurance, and promoting salt and fluid intake are indicated for patients with vasovagal } \\
\text { syncope, unless contraindicated. }\end{array}$ & I & $\mathrm{E}$ \\
\hline $\begin{array}{l}\text { Reducing or withdrawing medications that can cause hypotension can be beneficial for patients with } \\
\text { vasovagal syncope. }\end{array}$ & Ila & $\mathrm{E}$ \\
\hline $\begin{array}{l}\text { Physical counterpressure maneuvers can be useful for patients with vasovagal syncope who have a } \\
\text { sufficiently long prodromal period. }\end{array}$ & Ila & B-R \\
\hline $\begin{array}{l}\text { The use of fludrocortisone seems reasonable for patients with frequent vasovagal syncope who lack } \\
\text { contraindications for its use. }\end{array}$ & $\mathrm{llb}$ & $\mathrm{E}$ \\
\hline Beta-blockers may be considered for patients older than 40 years with frequent vasovagal syncope. & Illb & B-R \\
\hline $\begin{array}{l}\text { The use of midodrine seems reasonable for patients with frequent vasovagal syncope and no } \\
\text { hypertension or urinary retention. }\end{array}$ & $\mathrm{llb}$ & B-R \\
\hline \multicolumn{3}{|l|}{ Pacemakers for Syncope } \\
\hline $\begin{array}{l}\text { Dual-chamber pacing can be effective for patients } 40 \text { years of age or older with recurrent and } \\
\text { unpredictable syncope who have a documented pause } \geq 3 \text { seconds during clinical syncope or an } \\
\text { asymptomatic pause } \geq 6 \text { seconds. }\end{array}$ & Ila & B-R \\
\hline $\begin{array}{l}\text { Tilt-table testing may be considered to identify patients with a hypotensive response who would be less } \\
\text { likely to respond to permanent cardiac pacing. }\end{array}$ & llb & B-NR \\
\hline $\begin{array}{l}\text { Dual chamber pacing may be considered in adenosine-susceptible older patients who have unexplained } \\
\text { syncope without a prodrome, a normal ECG, and no structural heart disease }\end{array}$ & $\mathrm{llb}$ & C \\
\hline
\end{tabular}

trials and short- term controlled trials, the results of long-term, placebo controlled prospective trials have been less encouraging.

\section{Patient education}

The first step in the treatment of patients with VVS is an informative discussion with the patient about the benign nature and good prognosis of this disorder. Patients should be motivated to identify and avoid triggers of VVS, such as a hot environment, prolonged standing, and reduced water intake. Promotion of salt and fluid intake to expand circulating blood volume and increase venous return is encouraged unless contraindicated. Furthermore, discontinuation of hypotensive drug treatment for concomitant conditions is an important measure for the prevention of recurrent vasovagal syncope, especially in older patients.

\section{Physical counter pressure maneuvers}

Maneuvers such as hand-grip and leg crossing may inhibit vasovagal syncope by increasing the venous return. In one randomized prospective clinical trial, a physical counter pressure maneuver was superior to conventional therapy with a relative risk reduction of $39 \%$. Conventional therapy included patient education with the explanation of the mechanisms underlying vasovagal syncope and advice with regard to lifestyle modification (i.e., avoidance of triggers, lying down in case of symptoms, and increasing fluid and salt intake) [9]. Although the study was limited by its open-label design, given the benign nature of the intervention, physical counter pressure maneuvers should be part of the core management of patients with vasovagal syncope. 


\section{Beta-blockers}

These drugs have not been found to be effective in well-designed randomized, controlled studies. The largest multi-centred prospective, placebo-controlled, randomized control trial of beta-blocker therapy was the Prevention of Syncope (POST) trial, in which metoprolol was compared to placebo in patients with tilt positive presumed vasovagal syncope [10]. No overall benefit was found. Interestingly, in a metaanalysis of a large observational cohort and a prespecified substudy of POST, there was a statistically significant difference in response in those VVS patients aged $<42$ years and $\geq 42$ years (test of interaction $P=$ 0.007 ) [11]. The meta-analysis demonstrated a hazard ratio for recurrent syncope if treated with $\beta$-blockers of 1.58 (95\% CI, 1.00 to 2.31) for patients aged $<42$ years (placebo as better), and the hazard ratio was 0.52 (95\% CI, 0.27 to 1.01 ) for patients aged $\geq 42$ years (strong trend to benefit with metoprolol). Accordingly, $\beta$-blockers could be considered in older patients with VVS, but should be avoided in younger patients. The benefits of metoprolol in older patients with VVS are actively being assessed in the ongoing POST5 randomized clinical trial (NCT02123056, https://clinicaltrials.gov/ct2/show/ NCT02123056).

\section{Fludrocortisone}

Fludrocortisone is a synthetic mineralocorticoid that expands plasma volume and augments peripheral vasoconstriction through sensitization of peripheral $\alpha$-adrenergic receptors. The POST2 randomized clinical trial comparing fludrocortisone to placebo for vasovagal syncope (NCT00118482, https://clinicaltrials.gov/ show/ NCT00118482) was recently completed [12]. Although the study did not meet its primary outcome in reducing the likelihood of vasovagal syncope by the specified risk reduction of $40 \%$, when the analysis was restricted to outcomes after 2 weeks of dose stabilization, fludrocortisone significantly reduced the likelihood of syncope (HR 0.51; 95\% CI, 0.28 to 0.89; $p \leq 0.019$ ). Although the HRS consensus document predates the publication of POST2 results, the document states that fludrocortisone may be considered in patients whose symptoms severity warrants its use.

\section{Midodrine}

Peripheral alpha agonists, such as midodrine, may theoretically reduce venous pooling and counterbalance the reflex-mediated arterial vasodilation to prevent vasovagal syncope. In a meta-analysis of five randomized trials of midodrine, there was a risk difference of $37 \%$ (95\% CI, 21\% to 47\%) [13]. However, due to issues with study design and limited patient selection, these studies do not provide high-level evidence for midodrine use in adults. The utility of midodrine for patients is being assessed in the ongoing POST4 randomized controlled trial (NCT01456481, https://clinicaltrials.gov/show/ NCT01456481). In the current absence of compelling evidence, the HRS consensus document suggests that it may be reasonable to consider midodrine in patients whose symptoms severity warrants its use. Limitations of midodrine include its frequent dosing due to the short half-life, increased supine hypertension, its potential for urinary retention in men, and uncertainty regarding its potential teratogenic effects.

\section{Pacemakers}

Cardiac pacing has a very limited role in patients with typical vasovagal syncope. Early randomized trials of permanent pacing that demonstrated benefit had significant methodologic shortcomings that limit clinical application of their results. The Second Vasovagal Pacemaker Study (VPSII) and the Vasovagal Syncope and Pacing (SYNPACE) trial were the first doubleblind randomized, controlled trials of permanent pacing in vasovagal syncope designed to address these limitations. All patients received pacemakers, with the devices in the control arm programmed to only sense and not pace. In contrast to the open label trials, there was no significant difference in the rate of recurrent syncope over a 6-month follow up period [14,15].

The HRS consensus document does recommend pacing in highly selected patients - patients over 40 years of age, affected by frequent recurrences associated with frequent injury, limited prodrome, and documented asystole. This recommendation was made on the basis of the Third International Study on Syncope of Uncertain Etiology (ISSUE-3), which is the only positive doubleblind, randomized, placebo-controlled trial of pacing in patients with recurrent VVS [7]. There was a 57\% relative risk reduction $(\mathrm{P}<0.05)$ for recurrent syncope in the arm with the pacemaker switched on, observed over 2 years of follow up. It is worth noting that only $9 \%$ of vasovagal syncope referrals in the study qualified for randomization based on the ISSUE-3 inclusion criteria. While pacemakers have a role in the management of VVS, they should only be considered in highly selected patients.

\section{Conclusion}

Although syncope is a common problem, its management remains suboptimal. Over the past few decades, studies to better understand the natural history, risk stratification, and treatment have been hindered by lack of consistent syncope definitions that are applicable to a diverse group of healthcare providers. Fortunately, there has been a recent movement towards increased collaborative work between the various medical 
specialities that interact with syncope patients. The Heart Rhythm Society's 2015 Consensus Document identifies significant gaps in our understanding of vasovagal syncope in terms of their underlying causes, diagnostic challenges and management. It also highlights the need for well-conducted collaboration in the form of multi-centre studies through research networks and international registries. These ongoing collaborative efforts offer hope for future improvements in the care of patients with VVS.

\section{References}

1. Sheldon R, Killam S. Methodology of isoproterenol-tilt table testing in patients with syncope. J. Am. Coll. Cardiol. 19, 773779 (1992).

2. Brignole M, Alboni P, Benditt DG, et al. Guidelines on management (diagnosis and treatment) of syncope-- update 2004. Europace. 6, 467-537 (2004).

3. Moya A, Sutton R, Ammirati F, et al. Guidelines for the diagnosis and management of syncope (version 2009). Eur. Heart. J. 30, 2631-2671 (2009).

4. Sun BC, Costantino G, Barbic F, et al. Priorities for emergency department syncope research. Ann. Emerg. Med. 64, 649-655 (2014).

5. Sheldon RS, Grubb BP 2nd, Olshansky B, et al. 2015 heart rhythm society expert consensus statement on the diagnosis and treatment of postural tachycardia syndrome, inappropriate sinus tachycardia, and vasovagal syncope. Heart. Rhythm. 12, e41-63 (2015).

6. Sheldon R. How to Differentiate Syncope from Seizure. Cardiol. Clin. 33, 377-385 (2015).

7. Brignole M, Menozzi C, Moya A, et al. Pacemaker therapy in patients with neurally mediated syncope and documented asystole: Third International Study on Syncope of Uncertain Etiology (ISSUE-3): a randomized trial. Circulation. 125, 2566-2571 (2012).

8. Sutton R, Ungar A, Sgobino P, et al. Cardiac pacing in patients with neurally mediated syncope and documented asystole: effectiveness analysis from the Third International

\section{Disclosures}

SRR serves on the Steering Committee of the ADMIRE-ICD study and as a consultant to GE Healthcare, as a consultant to Lundbeck Pharmaceuticals, and he has received research support from Medtronic Inc., Bayer Inc., Dysautonomia International, the Canadian Institutes of Health Research, and the National Institutes of Health. RSS has received research funding from the Canadian Institutes of Health Research and the Canadian Arrhythmia Network of Canada. DC has no disclosures.

Study on Syncope of Uncertain Etiology (ISSUE-3) Registry. Europace. 16, 595-599 (2014).

9. van Dijk N, Quartieri F, Blanc JJ, et al. Effectiveness of physical counterpressure maneuvers in preventing vasovagal syncope: the Physical Counterpressure Manoeuvres Trial (PCTrial). J. Am. Coll. Cardiol. 48, 1652-1657 (2006).

10. Sheldon R, Connolly S, Rose S, et al. Prevention of Syncope Trial (POST): a randomized, placebo-controlled study of metoprolol in the prevention of vasovagal syncope. Circulation. 113, 1164-1170 (2006).

11. Sheldon RS, Morillo CA, Klingenheben T, et al. Agedependent effect of beta-blockers in preventing vasovagal syncope. Circulation. Arrhythmia and electrophysiology. 5, 920926 (2012).

12. Sheldon R, Raj SR, Rose MS, et al. Fludrocortisone for the Prevention of Vasovagal Syncope: A Randomized, PlaceboControlled Trial. J. Am. Coll. Cardiol. 68: 1-9 (2016).

13. Izcovich A, González Malla C, Manzotti M, Catalano HN, Guyatt G. Midodrine for orthostatic hypotension and recurrent reflex syncope: A systematic review. Neurology. 83, 1170-1177 (2014).

14. Connolly SJ, Sheldon R, Thorpe KE, et al. Pacemaker therapy for prevention of syncope in patients with recurrent severe vasovagal syncope: Second Vasovagal Pacemaker Study (VPS II): a randomized trial. JAMA. 289, 2224-2229 (2003).

15. Raviele A, Giada F, Menozzi C, et al. A randomized, doubleblind, placebo-controlled study of permanent cardiac pacing for the treatment of recurrent tilt-induced vasovagal syncope. The vasovagal syncope and pacing trial (SYNPACE). Eur. Heart. J. 25, 1741-1748 (2004). 